\title{
Phosphoinositide metabolism, lithium and manic depressive illness
}

\author{
Aysegul Yildiz \\ Dokuz Eylul Medical School, Department of Psychiatry, Izmir, Turkey \\ Tel.: + 90232244 4724; Fax: + 90232259 9723; E-mail: agul_yildiz@hotmail.com
}

\begin{abstract}
Physiology underlying manic depressive illness and treating effects of its most commonly used remedy - "lithium" is yet to be elucidated. Recent years of psychopharmacology research witnessed sparkling developments in our understanding of the mechanisms underlying lithium's mood stabilizing effects. Recent data on molecular biology and in vivo magnetic resonance spectroscopy suggest that some of the initial actions of lithium may occur through the inhibition of the enzyme inositol monophosphatase (IMPase) and reduction of myo-inositol, which in turn initiate a cascade of events at different levels of signal transduction process and gene expression in brain; such as the effects on protein kinase $\mathrm{C}$, myristoylated alenine rich C kinase substrate protein, glycogen synthase kinase $3 \beta$, B cell lymphoma-2 protein, and activator protein-I. It is likely that the enzyme IMPase other that being the key point in initiating lithium's therapeutic effects, may also play a critical role in the physiology underlying manic depressive illness.
\end{abstract}

Keywords: Lithium, phosphoinositide system, manic depressive illness

\section{Introduction}

Bipolar disorder is a chronic, disabling mental illness that affects at least 1-2\% (in recent studies it has been reported as high as 5.5 to $8.3 \%$ ) of the adult population and is associated with a substantial risk of suicide among those affected [1,8,10,45]. Since its discovery in 1949, lithium has become the most commonly and successfully used treatment for bipolar disorder [5,26]. Despite the important impact that lithium has made on the lives of millions affected by this illness, the cellular and molecular basis for its mood stabilizing effects has yet to be elucidated.

The most widely accepted hypothetical mechanism of action for lithium proposes that when lithium inhibits the enzyme inositol monophosphatase (IMPase); phosphoinositides and second messengers derived from their hydrolysis are depleted due to inositol deficiency $[3,4]$. However, in vivo data on lithium's inhibitory effect on the enzyme IMPase is limited and inconsistent [19,30,32,40,41]. Moreover, in recent years the validity of "inositol depletion hypothesis" has been called into question with some remarkable criticisms [2,16]. In this article, first we summarized available data on the lithium's effect on the enzyme IMPase in vivo. Second, we discussed the validity of the "inositol depletion" hypothesis in the light of recent criticisms. Third, we provided a brief summary of the recent research data on the molecular mechanisms underlying therapeutic effects of lithium with their relevance to the lithium-induced reductions in myo-inositol levels. Finally, we made an evidence based discussion on the enzyme IMPase as a potential cornerstone in the pathophysiology of manic depressive illness. 


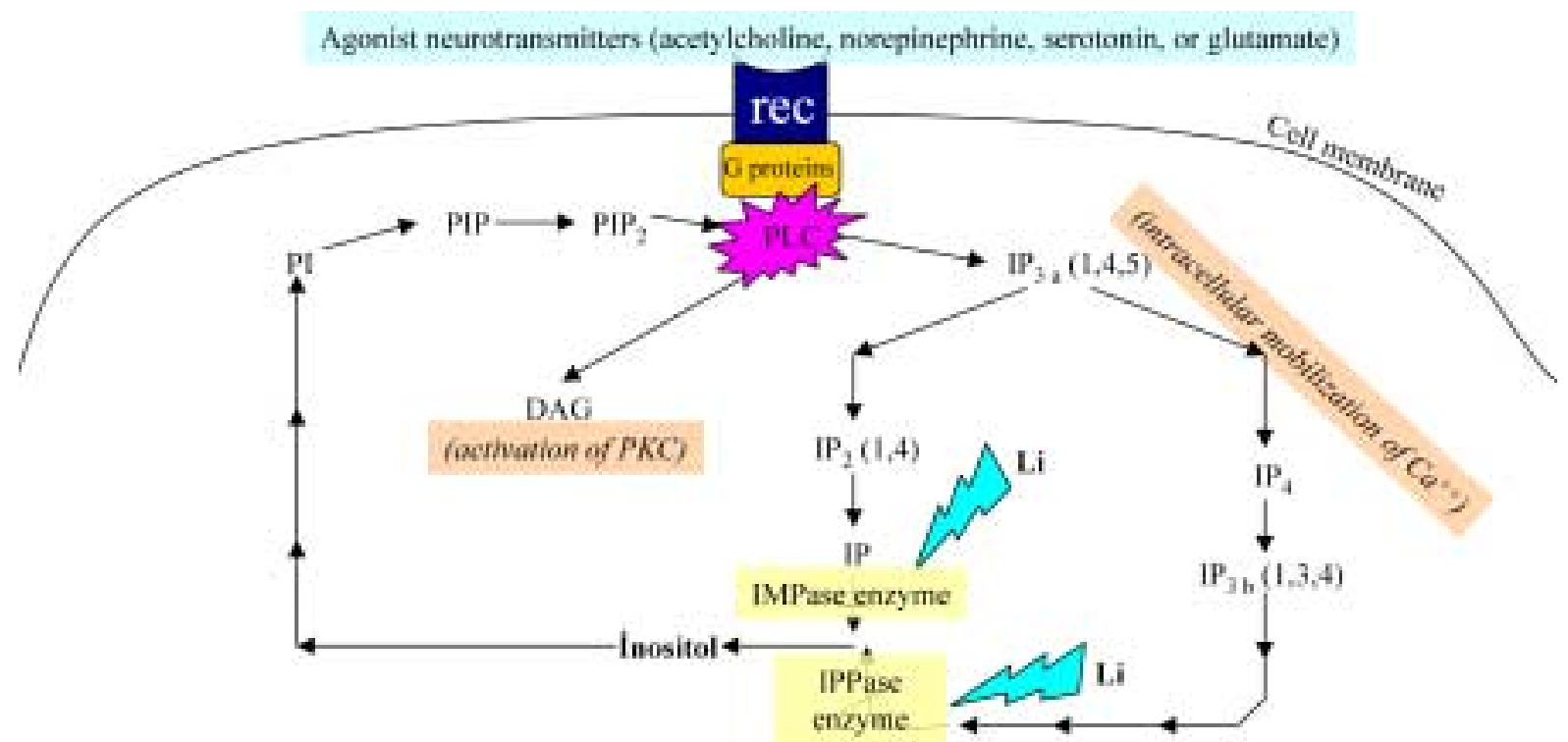

Fig. 1. Effect of lithium on phosphoinositide cycle. Abbreviations: PLC, phospholipase C; PIP ${ }_{2}$, phosphatidylinositol-4,5-biphosphate; $\mathrm{IP}_{3 \mathrm{a}}$, inositol-1,4,5-triphosphate; DAG, diacylglycerol; $\mathrm{IP}_{4}$, inositol-1,3,4,5-tetraphosphate; IP $\mathrm{P}_{3 \mathrm{~b}}$, inositol-1,3,4-triphosphate; IP 2 , inositol-1,4-biphosphate; IP, inositol-1-monophosphate; PI, fosfatidylinositol; PIP, fosfatidyinositolphosphate, IMPase enzyme, inositol monophosphatase enzyme; IPPase enzyme, inositol poliphophate-1-phophatase enzyme. Adapted from [48].

\section{In vivo data on the lithium's inhibitory effect on the enzyme IMPase}

Neurotransmitters such as acetylcholine, norepinephrine, serotonin, and glutamate bind to specific cell surface receptors, which interact with $G$ proteins [26]. These proteins stimulate the membrane enzyme, phospholipase C (PLC) [26]. Activated PLC catalyzes the conversion of phosphatidylinositol-4,5biphosphate $\left(\mathrm{PIP}_{2}\right)$ to the second messengers, inositol-1,4,5-triphosphate $\left(\mathrm{IP}_{3}\right)$ and diacylglycerol (DAG) [26]. $\mathrm{IP}_{3}$ stimulates the mobilization of intracellular $\mathrm{Ca}^{++}$, while DAG activates protein kinase-C (PKC) [26]. $\mathrm{IP}_{3}$ can be phosphorylated or dephosphorylated, leading to other inositol phosphate compounds or to unphosphorylated inositol. Inositol may be converted to phoshatidylinositol (PI), which in turn is phosphorylated to phosphatidylinositolphosphate (PIP) and PIP ${ }_{2}$, and recycled (Fig. 1) [26].

Since, brain myo-inositol is derived primarily from recycling of inositol phosphates, sufficient supplies of myo-inositol is crucial to a cell for the maintenance and efficacy of signaling [26]. Lithium, at therapeutically relevant concentrations, is an uncompetitive inhibitor of IMPase $\left(K_{i}=0.8 \mathrm{mM}\right)$, and lithium administration is assumed to result in an accumulation of inositol-1-monophosphate (I1P), as well as a reduction in free inositol [26,37]. Lithium also inhibits inositol polyphosphate-1-phosphatase (IPP), which is involved in recycling inositol phosphates to inositol (Fig. 1) [26,37]. Thus, it has been hypothesized that a physiologic consequence of lithium's action is derived through a depletion of free myo-inositol in the brain [26].

However, data relating effects of lithium on receptor mediated phosphoinositide responses are inconsistent and are limited by considerable methodological variability. Therefore, many questions remain regarding the experimental basis of the "inositol depletion" hypothesis, and its applicability to in vivo response to lithium administration [16]. Considering the lack of animal models of bipolar illness, species selectivity of the pharmacological effects of psychotrophic drugs [16,38], and lithium's unique effects 
on the brain, Magnetic Resonance Spectroscopy (MRS) offers a valuable tool to investigate the validity of the "inositol depletion" hypothesis in vivo human brain.

The effect of lithium on IMPase activity may be investigated using either proton $\left({ }^{1} \mathrm{H}\right) \mathrm{MRS}$, which has the potential to detect myo-inositol (the product of the enzyme), or phosphorous $\left({ }^{31} \mathrm{P}\right) \mathrm{MRS}$, which can estimate brain phosphomonoesters (PME), which include inositol-1-monophosphate (the substrate of the enzyme) $[11,18]$. When the enzyme IMPase is inhibited by lithium, myo-inositol levels would be expected to decrease while the substrate inositol-1-monophosphate would be expected to increase. However, direct in vivo measurement of myo-inositol level is not possible. With ${ }^{1} \mathrm{H}$ MRS, the myo-inositol resonance has contributions from three metabolites that can be affected by altered lithium concentrations in vivo, namely myo-inositol, inositol-1-monophosphate, and glycine. Therefore, the decrease in myo-inositol levels may be obscured by an increase in inositol-1-monophosphate and glycine levels [40].

A few human MRS studies have investigated the effect of lithium on the enzyme IMPase. Using ${ }^{1} \mathrm{H}$ MRS, Moore and associates (1999) documented a lithium-induced decrease in myo-inositol concentrations in the right frontal lobe of bipolar patients [30]. Although subject to a type II error by using ${ }^{31} \mathrm{P}$ MRS, Kato et al. (1993), found no change in PME concentrations in 6 manic patients, examined before and after initiation of lithium [19]. Another study utilizing ${ }^{31} \mathrm{P}$ MRS found that in 21 psychotic patients given lithium for two weeks, there were no changes in PME concentrations [32]. Silverstone and associates $(1996,1999)$ did not observe any significant effects of 7 days of lithium administration either on PME ratios or on myo-inositol levels in temporal lobe of healthy volunteers [40,41]. However, they reported a significant increase on the PME ratios after the stimulation of the phosphoinositide cycle by amphetamine [41].

In ${ }^{31} \mathrm{P}$ MR spectra of brain, typically seven peaks are resolved; PME, inorganic phosphate $\left(\mathrm{P}_{\mathrm{i}}\right)$, phosphodiesters (PDE), phosphocreatine (PCr), as well as $\gamma, \alpha$, and $\beta$ nucleotide triphosphate (NTP), (Fig. 2). The PME peak contains signals from several metabolites, including metabolites related to membrane phospholipid synthesis, such as phosphocholine, phosphoethanolamine, and sugar phosphates such as $\alpha$-glycerophosphate and inositol phosphates, including I1P [11,18]. It is estimated that inositol monophosphates constitute only about $10-15 \%$ of the total PME peak area [36]. Under normal circumstances, the concentration of inositol-1-phosphate is $0.05-0.1 \mathrm{mmol} / \mathrm{l}$, whereas with ${ }^{31} \mathrm{P}$ MRS the minimum concentration for detection is $0.5-1 \mathrm{mmol} / 1[11,43]$. Thus, a 5 - to 10 -fold increase in I1P concentration would be necessary for detection as an increase in the PME resonance [43].

There are a limited number of ways by which the sensitivity of ${ }^{31} \mathrm{P}$ MRS experiments may be increased. In particular, proton decoupling [18,31] and increasing the volume of tissue examined are the easiest methods to implement on an existing scanner (Fig. 2). With proton decoupling, protons that interact with phosphorous are irradiated to improve the spectral resolution deteriorated by spin-spin coupling [18]. Relying on that we collected proton decoupled ${ }^{31} \mathrm{P}$ MR spectra from healthy human subjects before and after the administration of lithium on a 1.5 T GE SIGNA MR system equipped for broadband spectroscopy [46]. Our a priori hypothesis was that the decoupled ${ }^{31} \mathrm{P}$ MR spectra obtained from a large volume of brain would reveal increases in the PME concentration after the chronic administration of lithium. 8 subjects, 4 women, and 4 men completed the full 14 days of the study period. With pair-wise comparisons of the mole percent PME values, we detected a significant increase following chronic lithium administration both on day $7\left(p<0.04, t_{\mathrm{df}=7}=2.57\right.$, standard deviation $(\mathrm{SD})$ of change $\left.=1.67\right)$, and also on day 14 compared to baseline $\left(p<0.04, t_{\mathrm{df}=7}=2.58, \mathrm{SD}_{\text {change }}=1.34\right)$ [46].

In this longitudinal study we have shown that lithium treatment increases PME levels in human brain [46]. This increase was observed at the $1_{\text {st }}$ week and maintained through the 2 nd week of lithium administration [46]. Prior studies showing an increase in the PME peak following lithium administration 


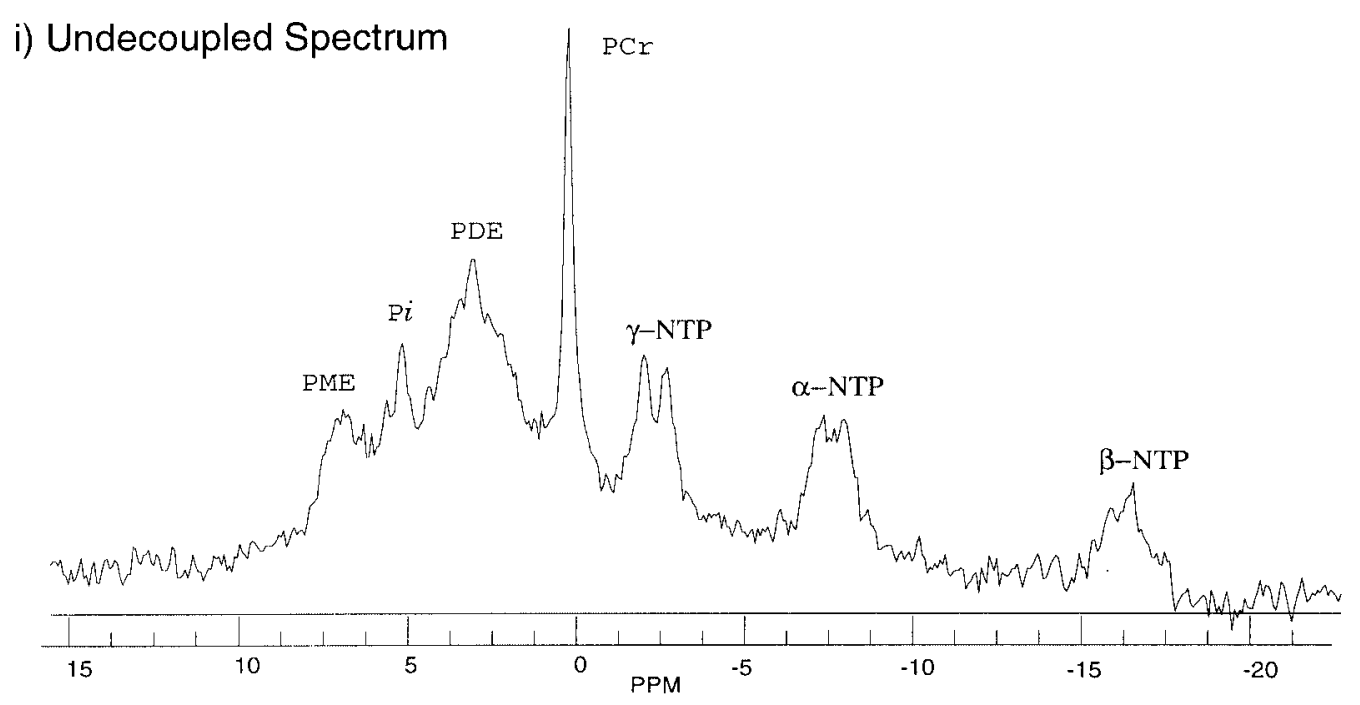

ii) Decoupled Spectrum

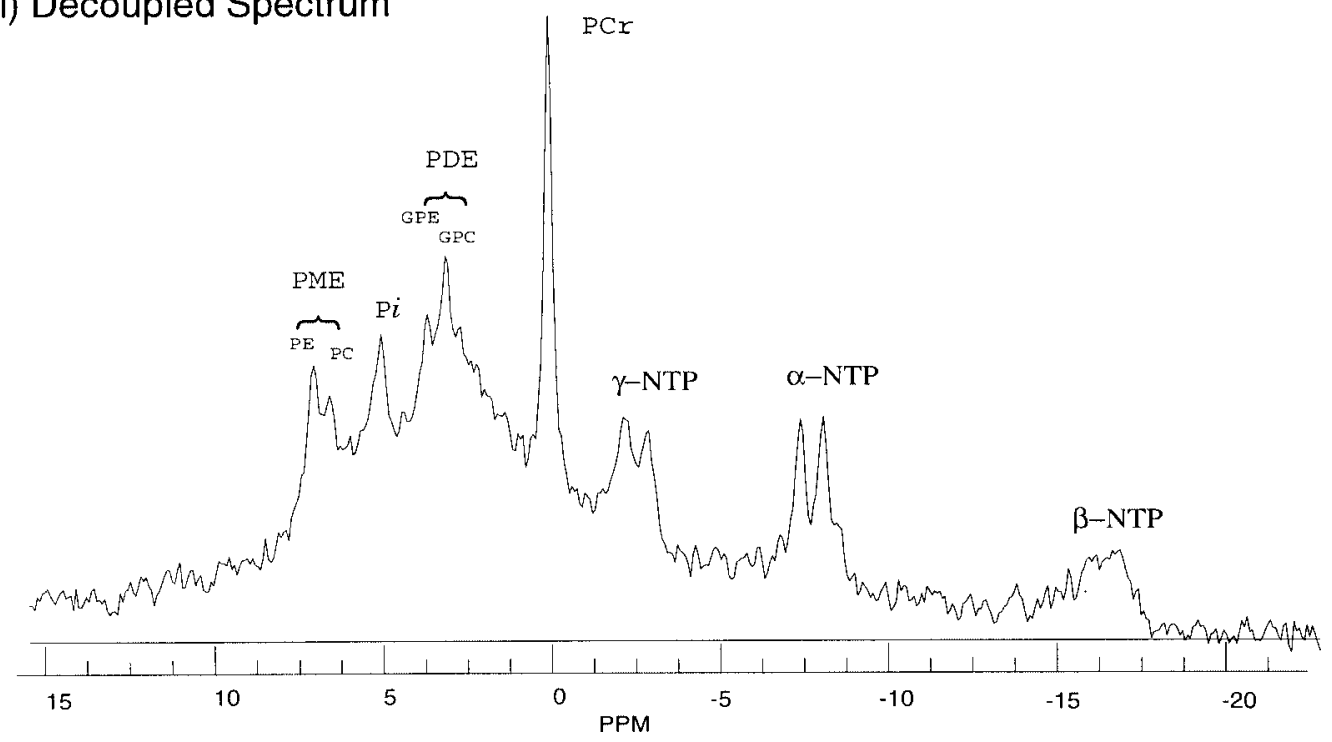

Fig. 2. Typical ${ }^{31} \mathrm{P}-\mathrm{MR}$ spectra: (i) undecoupled, (ii) decoupled. Frequency units are expressed in parts per million (PPM). PME, phosphomonoesters; PE, phosphoethanolamine; PC, phosphocholine; Pi, inorganic phosphate; PDE, phosphodiesters; GPE, glycerophosphoethanolamine; GPC, glycerophosphocholine; PCr, phosphocreatine; $\gamma, \alpha$, and $\beta$-NTP, gamma, alpha, and beta nucleotidetriphosphates. Adapted from [46].

to cats and rats, using in vitro MRS, determined that this change was due to increases of inositol-1monophosphate concentrations [34-36]. Another in vivo study showed that chronic lithium administration caused a significant decrease in platelet membrane phosphatidyl inositol-4,5-biphosphate levels in bipolar subjects [42]. Although, these findings provide evidence for the inositol-depleting effects of lithium, it should be considered that inositol depletion per se may not be responsible for lithium's therapeutic effects. 


\section{Validity of the "inositol depletion" hypothesis has been called into question}

While data from preclinical studies have tended to demonstrate lithium-induced alterations in receptormediated phosphoinositide turnover, numerous methodologic criticisms have been raised recently, and the validity of the inositol depletion hypothesis, as originally articulated, has been called into question $[2,16]$. One methodologic criticism is that the inositol depleting effect of lithium observed in some cases in brain slices is dependent on the inositol-depleted state of the slices (inositol is already depleted by about $80 \%$ in the absence of lithium in washed rat brain slices). In addition, lithium's effects on IMPase are species-selective and can not be extrapolated to in vivo human brain $[2,16]$. Another major criticism is that with therapeutic concentrations of lithium, only partial inhibition of IMPase will be obtained and, even with toxic doses, lithium causes modest reductions in inositol concentrations (about 35\% at most). It is unknown if such a modest decrease of brain inositol is sufficient to limit phosphoinositide synthesis and impair signal transduction $[2,16]$. Thus, inositol depletion by itself may not be responsible for lithium's therapeutic effects $[28,29]$. Instead, as some preclinical $[9,21,44]$ and clinical data $[30,46]$ suggest, some of the initial actions of lithium may occur through a reduction of myo-inositol, which in turn may initiate a cascade of secondary changes at different levels of signal transduction process and gene expression in brain, effects that are ultimately responsible for the anti-manic/mood stabilizing and neuroprotective effects of lithium $[15,16,28,29]$. Consistent with this model, recent findings in molecular biology established some remarkable effects of lithium at various levels of cellular function in brain [26].

\section{The cascade of secondary changes presumably initiated by the lithium-induced reduction of myo-inositol}

It has recently been demonstrated that lithium provoke some significant changes in signal transduction systems and gene expression in brain.

\subsection{G proteins \& lithium}

Lithium is known to affect two major second messenger generating systems in the brain, namely the phosphoinositide turnover and adenylyl cyclase activity, which generates cyclic adenosine monophosphate (cAMP) [26]. Since, both of these systems are connected to cell surface receptors, and lithium affects these systems without influencing receptor levels per se, it has been considered that lithiums' effects are mediated through the $G$ proteins that are directly connected to receptors [26].

Recent findings suggest that lithium modifies $G$ protein function without effecting $G$ protein levels [26]. It has been shown that lithium produce a significant increase in pertussis toxin-catalyzed adenine nucleotide diphosphate (ADP)-ribosylation in platelets of subjects receiving lithium treatment [13, 26]. Pertussis toxin selectively catalyzes the ADP-ribosylation of the undissociated, inactive $\alpha \beta \gamma$ heterotrimeric form of inhibitory $G$ protein $\left(G_{i}\right)$. Thus, this finding indicates that lithium inactivates $G_{i}$ by stabilizing the inactive conformation $[23,26]$. It has also been shown that chronic lithium causes an enhancement of both basal and post-receptor stimulated adenylyl cyclase activity in human platelet preparations and rat brain. It is likely that this effect of lithium on adenylyl cyclase activity occurs as a consequence of the suppression of the tonic inhibitory control of G protein (Fig. 3) [23]. 


\subsection{Gene expression \& lithium}

It is widely accepted that drugs, which require a lag period for onset of action and are not immediately reversed upon discontinuation such as the majority of the psychotropic medications, must exert their effects at the genomic level $[15,26]$.

Activator protein-1 (AP-1), is a complex composed of products from two transcription factor families; "fos" and "jun". These products bind to a common DNA site in the regulatory domain of the gene, namely "12-o-tetradecanoyl-phorbol 13-acetate (TPA) response element-TRE" and activate gene transcription in response to protein kinase C (PK C) activators, growth factors, cytokines, and neurotransmitters [14,17]. It has been demonstrated that lithium at therapeutic concentrations produce a time-dependent increase in the DNA binding of AP-1 to TRE [6,33]; and this effect translates into alterations at the gene expression level $[6,33,49]$.

It is yet to be delineated how lithium regulates AP-1 DNA binding, but may involve effects on mitogenactivated protein kinases. Supportively, lithium at therapeutic doses has been shown to inhibit the activity of glycogen synthase kinase- $3 \beta(\mathrm{GSK}-3 \beta)$, which is known to phosphorylate c-jun at 3 sites adjacent to the DNA binding domain, by this means reducing TRE binding $[12,20]$. In view of that, lithium by means of lessening GSK-3 $\beta$ activity may be enhancing AP-1 binding to the TRE, (Fig. 3) [48].

\subsection{Protein kinase $C \&$ lithium}

Protein kinase $\mathrm{C}$ family consists of at least 12 phosphorylating isozymes that are distributed heterogeneously in the brain. Each isozyme has different substrate affinities and second messenger activators suggesting distinct cellular functions [23].

It has been demonstrated that chronic lithium administration results in significant decrease in membrane-associated PK C- $\alpha$ and $-\varepsilon$ isoforms [27]. PK C is a calcium activated diacylglyceroldependent protein kinase and is known to be involved in regulation of signal transduction processes, ion channels' function and gene transcription in cell [24]. Besides, it has been shown that lithium induced down-regulation of PK C- $\alpha$ and $-\varepsilon$ can be reversed by coadministration of myo-inositol in vivo, a finding that is confirming the hypothesis that lithium induced changes at different levels of signal transduction processes and gene expression may be secondary to lithium induced reductions in myo-inositol levels in brain.

Lenox and colleagues (1992) have revealed a decrease in myrstoylated alenine-rich C kinase substrate (MARCKS) by chronic administration of lithium [25]. MARCKS is protein kinase $\mathrm{C}$ substrate in brain and regulates cellular localization and function of PK C in cell through phosphorylation. MARCKS binds calmodulin in a calcium dependent manner and cross links actin at plasma membrane. Thus, MARCKS has been implicated in cellular processes associated with cytoskeletal restructuring and long-term neuroplastic changes. It has been shown that coadministration of myo-inositol in vivo, also reverses lithiuminduced decrease in MARCKS, again supporting the hypothesis that myo-inositol reduction constitutes the initial step in lithium-induced alterations in brain (Fig. 3) [24].

\subsection{B cell lymphoma protein-2 \& lithium}

B cell lymphoma protein-2 (Bcl-2), is a neuroprotective protein which enhances the survival of cells when exposed to adverse stimuli [22]. Chen and associates (1999) has found that chronic lithium at therapeutically relevant doses increases messenger RNA levels of the polyoma-virus enhancer-binding 


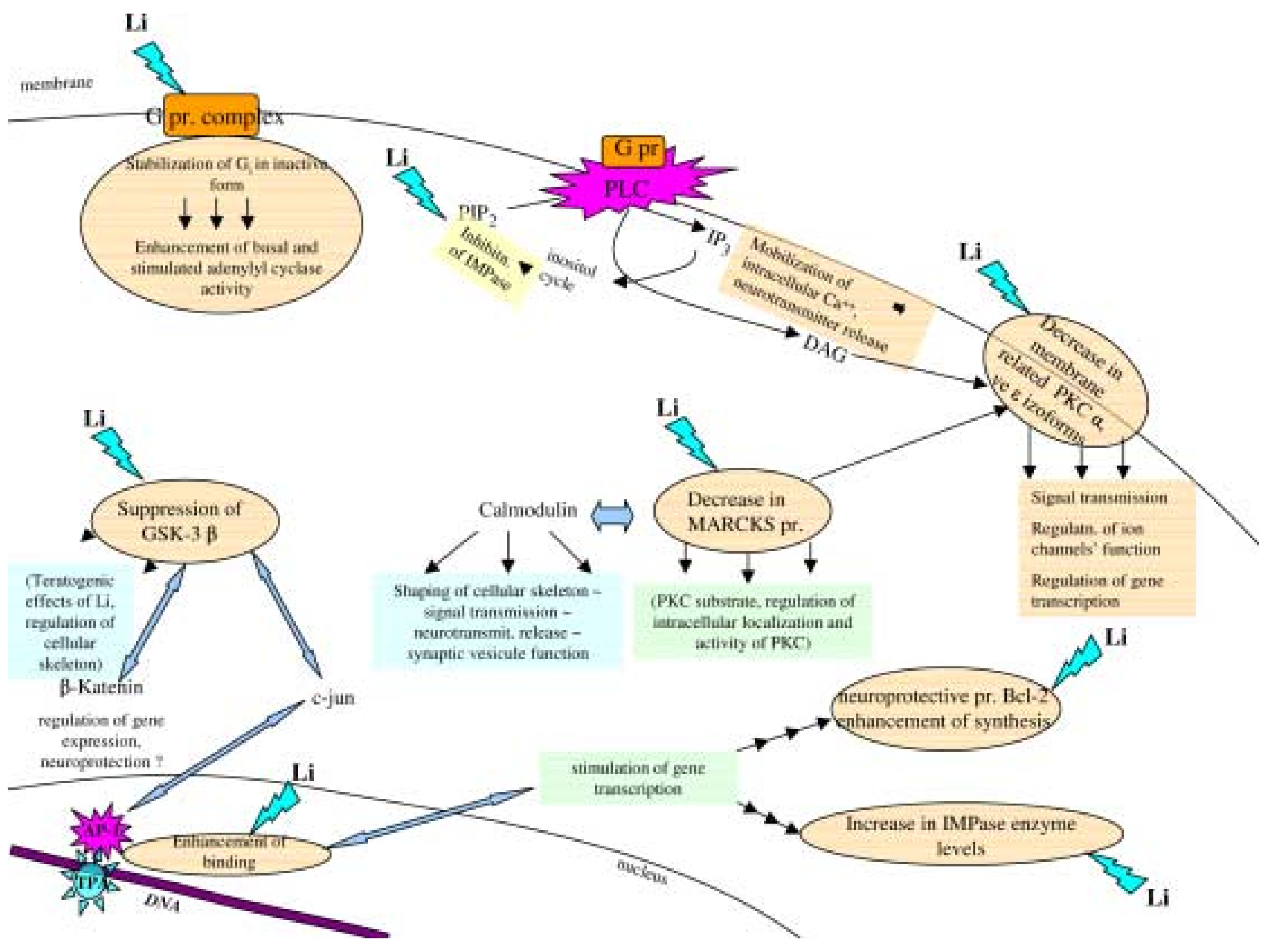

Fig. 3. Effects of lithium at different levels of cellular functions. Abbreviations: $\mathrm{G}_{\mathrm{i}}$, inhibituar $\mathrm{G}$ protein; PLC, phospholipase C; PIP 2 phosphatidylinositol-4,5-

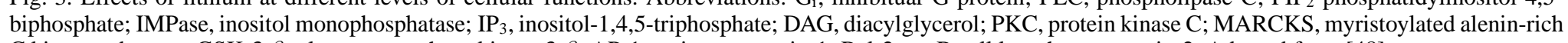
C kinase substrate; GSK-3 $\beta$, glycogen synthase kinase $3 \beta$; AP-1, activator protein-1; Bcl-2 pr., B cell lymphoma protein-2. Adapted from [48]. 
protein- $2 \beta$ (PEBP-2 $\beta$ ) transcription factors [7]. As, PEBP-2 $\beta$ regulates synthesis of $\mathrm{Bcl}-2$ in brain; lithium, by increasing PEBP-2 $\beta$ levels produces a robust increase in Bcl-2 in brain [7].

Obviously, last decade has witnessed enormous progress in our understanding of mechanisms underlying lithium's therapeutic effects in manic-depressive illness. There is no question that it is not as simple as originally suggested by the "inositol depletion hypothesis". Nonetheless, there still exist some gaps in connecting all these data together and puzzling out the mechanism underlying lithium's mood stabilizing effects in bipolar illness. Yet, today's knowledge on the matter points toward the hypothesis that a lithium-induced reduction in myo-inositol levels (through inhibition of the IMPase) may initiate a cascade of events at various levels of cellular function, which results in treating effects of lithium in manic-depressive illness (Fig. 3).

\section{If the enzyme IMPase is the key point in initiating lithium's therapeutic effects, could it be the case that it might also be involved in the pathophysiology of bipolar illness?}

Today's data suggest that the enzyme IMPase plays an important role for the function of lithium in bipolar patient's brain. If this is the case for the treatment effects of lithium in bipolar illness, we asked if this enzyme could be affected in bipolar patients. To investigate this we run a meta-analysis including all the available in vivo data of human brain, and compared the PME values of bipolar patients in different mood states to healthy controls [47]. In this work, we detected lower PME levels in bipolar euthymics in comparison to healthy controls $(p=0.014)$ [47]. In regard to the state dependent alterations, bipolar patients with current depressive episodes had higher PME levels than bipolar euthymics ( $p=0.0005)$; in manic vs. euthymic subjects however, we couldn't detect significance $(p=0.247)$ [47].

Since most of the subjects in the studies included in this meta-analysis were on lithium or off lithium for only one week; low PME levels in the euthymic and high PME levels in the depressive state of bipolar patients might be the reflections of lithium's long-term pharmacologic effects.

The PME resonance has contributions from the inositol-1-monophosphate [11,18]. Shamir et al. (1998) have reported that cell lines from bipolar patients have significantly lower inositol monophosphatase (IMPase) activity than cell lines from control subjects [39]. Besides, among bipolar patients, lithium responders exhibited significantly lower IMPase activity compared to nonresponders [39]. Lithium has been demonstrated to inhibit the enzyme IMPase, and increase PME in vivo human brain $[37,46]$. It is a heuristic hypothesis that bipolar subjects exhibit genetically determined low IMPase activity and lithium, by causing more inhibition of the enzyme activity, may cause an increase in inositol 1-monophosphate and/or a decrease in free inositol beyond a critical point which stimulates a compensatory transcriptional up-regulation of the enzyme's synthesis [47]. Supportively, it has been shown that chronic lithium administration causes an increase in IMPase and its messenger RNA levels [35,39]. When IMPase activity is increased, a bipolar patient's clinical status would be stabilized in euthymic state, and inositol 1-monophosphate levels as well as the PME levels would be decreased. Whenever the compensatory up regulating effect of lithium on the enzyme IMPase is lost, the patient's clinical status would be destabilized and the patient would have an episode of altered mood state [47]. In that case, since the enzyme is less active again, inositol-1-monophosphate levels and the PME levels would go up [47]. The results of our meta-analysis are compatible with this hypothesis for the eythymic and depressed states, but not for the manic state [47]. This may result from the inconsistency of the available data, and/or the small number of studies included in the meta-analysis of the PME in manic state.

In conclusion, lithium, by inhibiting the enzyme IMPase produce a reduction in myo-inositol and an increase in inositol-1-monophosphate levels. This initial decrease in myo-inositol or increase in I1P levels 
in turn initiates a cascade of secondary effects at various levels of signal transduction processes and gene expression in brain including effects of lithium on PK C, MARKS, GSK-3 $\beta$, and Bcl-2 proteins. Recent research data in molecular biology as well as in vivo MRS suggest that IMPase may have a critical role in the physiology underlying lithium's treating effects in bipolar illness as well as the disease process itself.

\section{References}

[1] J. Angst, The emerging epidemiology of hypomania and bipolar II disorder, J. Aff. Disord. 50 (1998), 143-151.

[2] J.R. Atack, Inositol monophosphatase, the putative therapeutic target for lithium, Brain Res. Rev. 22 (1996), 183-190.

[3] M.J. Berridge, C.P. Downes and M.R. Hanley, Lithium amplifies agonist-dependent phosphotidylinositol responses in brain and salivary glands, Biochem. J. 206 (1982), 587-595.

[4] M.J. Berridge, C.P. Downes and M.R. Hanley, Neural and developmental actions of lithium: a unifying hypothesis, Cell 59 (1989), 411-419.

[5] J. Cade, Lithium salts in the treatment of psychotic excitement, Med. J. Australia 2 (1949), 349-352.

[6] G. Chen, P.X. Yuan, D.B. Hawver, W.Z. Potter and H.K. Manji, Increase in AP-1 transcription factor DNA binding activity by valproic acid, Neuropsychopharmacology 16 (1997), 238-245.

[7] G. Chen, W.Z. Zeng, P.X. Yuan, L.D. Huang, Y.M. Jiang, Z.H. Zhao and H.K. Manji, The mood stabilizing agents lithium and valproate robustly increase the level of the neuroprotective protein bcl-2 in the CNS, J. Neurochem. 72 (1999), 879882.

[8] A. Coppen, H. Standish-Barry, J. Bailey, G. Houston, P. Silcocks and C. Hermon, Long-term lithium and mortality, Lancet 335 (1990), 1347.

[9] P.P. Godfrey, Potentiation by lithium of CMP-phosphatidate formation in carbachol-stimulated rat cerebral-cortical slices and its reversal by myo-inositol, Biochem. J. 258 (1989), 621-624.

[10] F.K. Goodwin and K.R. Jamison, Manic-Depressive Illness, Oxford University Press, New York, 1990.

[11] L. Gyulai, L. Bolinger, J.S. Leigh, C. Barlow and B. Chance, Phosphoethanolamine - the major constituent of the phosphomonoester peak observed by ${ }^{31} \mathrm{P}$ NMR on developing dog brain, FEBS Lett. 178 (1984), 137-142.

[12] C.M. Hedgepeth, L.J. Conrad, J. Zhang, H.C. Huang, V.M.Y. Lee and P.S. Klein, Activation of the Wnt signaling pathway: a molecular mechanism for lithium action, Dev. Biol. 185 (1997), 82-91.

[13] J.K. Hsiao, H.K. Manji, G.A. Chen, J.A. Bitran, E.D. Risby and W.Z. Potter, Lithium administration modulates platelet $\mathrm{G}_{\mathrm{i}}$ in humans, Life Sci. $\mathbf{5 0}$ (1992), 227-233.

[14] P. Hughes and M. Dragunow, Induction of immediate early genes and the control of neurotransmitter-regulated gene expression within the nervous system, Pharmacol. Rev. 47 (1995), 133-178.

[15] S.E. Hyman and E.J. Nestler, Initiation and adaptation: A paradigm for understanding psychotropic drug action, Am. $J$. Psychiatry 153 (1996), 151-162.

[16] R.S. Jope and M.B. Williams, Lithium and brain signal transduction systems, Biochem. Pharmacol. 47 (1994), 429-441.

[17] M. Karin and T. Smeal, Control of transcription factors by signal transduction pathways: the beginning of the end, Trends Biochem. Sci. 17 (1992), 418-422.

[18] T. Kato, T. Inubushi and N. Kato, Magnetic resonance spectroscopy in affective disorders, J. Neuropsyc. Clin. Neurosci. 10 (1998), 133-147.

[19] T. Kato, S. Takahashi, T. Shioiri and T. Inubushi, Alterations in brain phosphorous metabolism in bipolar disorder detected by in vivo ${ }^{31} \mathrm{P}$ and ${ }^{7} \mathrm{Li}$ magnetic resonance spectroscopy, J. Affective Dis. 27 (1993), 53-60.

[20] P.S. Klein and D.A. Melton, A molecular mechanism for the effects of lithium on development, Proc. Nat. Acad. Sci. USA 93 (1996), 8455-8459.

[21] O. Kofman and R.H. Belmaker, Biochemical, behavioral and clinical studies of the role of inositol in lithium treatment and depression, Biol. Psychiatry 34 (1993), 839-852.

[22] M.S. Lawrence, D.Y. Ho, G.H. Sun, G.K. Steinberg and R.M. Sapolsky, Overexpression of Bcl-2 with herpes simplex virus vectors protects CNS against neurological insults in vitro and in vivo, J. Neurosci. 16 (1996), 486-496.

[23] R.H. Lenox, R.K. McNamara, R.L. Papke and H.K. Manji, Neurobiology of lithium: An update, J. Clin. Psychiatry 59(Suppl. 6) (1998), 37-47.

[24] R.H. Lenox, R.K. McNamara, J.M. Watterson and D.G. Watson, Myristoylated alenine-rich C kinase substrate (MARCKS): A molecular target for the therapeutic action of mood stabilizers in the brain, J. Clin. Psychiatry 57(Suppl. 13) (1996), 23-31.

[25] R.H. Lenox, D.G. Watson, J. Patel and J. Ellis, Chronic lithium administration alters a prominent PKC substrate in rat hippocampus, Brain Res. 570 (1992), 333-340. 
[26] H.K. Manji, J.M. Bebchuk, G.J. Moore, D. Glitz, K.A. Hasanat and G. Chen, Modulation of CNS signal transduction pathways and gene expression by mood stabilizing agents: Therapeutic implications, J. Clin. Psychiatry 60(Suppl. 2) (1999), 27-39.

[27] H.K. Manji, G. Chen, J.K. Hsiao, E.D. Risby, M.I. Masana and W.Z. Potter, Regulation of signal transduction pathways by mood-stabilizing agents: Implications for delayed onset of therapeutic efficacy, J. Clin. Psychiatry 57(Suppl. 13) (1996), $34-46$.

[28] H.K. Manji and R.H. Lenox, Lithium: A molecular transducer of mood-stabilization in the treatment of bipolar disorder, Neuropsychopharmacology 19 (1998), 161-167.

[29] H.K. Manji, W.Z. Potter and R.H. Lenox, Signal transduction pathways: Molecular targets for lithium's actions, Arch. Gen. Psychiatry 52 (1995), 531-543.

[30] G.J. Moore, J.M. Bebchuk, J.K. Parrish, M.W. Faulk, C.L. Arfken, J. Strahl-Bevacqua and H.K. Manji, Temporal dissociation between lithium induced changes in frontal lobe myo-inositol and clinical response in manic-depressive illness, $A m$. J. Psychiatry 156 (1999), 1902-1908.

[31] J. Murphy-Boesch, R. Stoyanova, R. Srinivasan, T. Willard, D. Vigneron, S. Nelson, J.S. Taylor and T.R. Brown, Protondecoupled ${ }^{31} \mathrm{P}$ chemical shift imaging of the human brain in normal volunteers, NMR in Biomedicine 6 (1993), 173-180.

[32] H.A. Nasrallah and J.W. Pettegrew, NMR Spectroscopy in Psychiatric Brain Disorders, American Psychiatric Press, Progress in Psychiatry, Vol. 47, 1995, pp. 135-140.

[33] N. Ozaki and D.M. Chuang, Lithium increases transcription factor binding to AP-1 and cyclic AMP-response element in cultured neurons and rat brain, J. Neurochem. 69 (1997), 2336-2344.

[34] N.E. Preece, D.G. Gadian, J. Houseman and S.R. Williams, Lithium induced modulation of cerebral inositol phosphate metabolism in the rat: A multinuclear magnetic resonance study in vivo, Lithium 3 (1992), 287-297.

[35] P.F. Renshaw, N.E. Joseph and J.S. Leigh, Chronic dietary lithium induces increased levels of myo-inositol-1-phosphatase activity in rat cerebral cortex homogenates, Brain Res. 380 (1986), 401-404.

[36] P.F. Renshaw, M.D. Schnall and J.S. Leigh, In vivo ${ }^{31} \mathrm{P}$ NMR spectroscopy of agonist stimulated phosphatidylinositol metabolism in cat brain, Magn. Reson. Med. 4 (1987), 221-226.

[37] P.F. Renshaw, J.J. Summers, C.E. Renshaw, K.G. Hines and J.S. Jr. Leigh, Changes in the ${ }^{31}$ P NMR Spectra of cats receiving lithium chloride systemically, Biol. Psychiatry 21 (1986), 694-698.

[38] D.L. Rosin, K. Melia, A.M. Knorr, E.J. Nestler, R.H. Roth and R.S. Duman, Chronic imipramine administration alters the activity and phosphorylation state of tyrosine hydroxylase in dopaminergic regions of rat brain, Neuropsychopharmacology 12 (1995), 113-121.

[39] A. Shamir, R.P. Ebstein, L. Nemanov, A. Zohar, R.H. Belmaker and G. Agam, Inositol monophosphatase in immortalized lymphoblastoid cell lines indicates susceptibility to bipolar disorder and response to lithium therapy, Mol. Psychiatry 3 (1998), 481-482.

[40] P.H. Silverstone, C.C. Hanstock, J. Fabian, R. Staab and P.S. Allen, Chronic lithium does not alter human myo-inositol or phosphomonoester concentrations as measured by ${ }^{1} \mathrm{H}$ and ${ }^{31} \mathrm{P}$ MRS, Biol. Psychiatry 40 (1996), 235-246.

[41] P.H. Silverstone, S. Rotzinger, A. Pukhovsky and C.C. Hanstock, Effects of lithium and amphetamine on inositol metabolism in the human brain as measured by ${ }^{1} \mathrm{H}$ and ${ }^{31} \mathrm{P}$ MRS, Biol. Psychiatry 46 (1999), 1634-1641.

[42] J.C. Soares, A.G. Mallinger, C.S. Dippold, K. Forster Wells, E. Frank and D.J. Kupfer, Effects of lithium on platelet membrane phosphoinositides in bipolar disorder patients: A pilot study, Psychopharmacology 149 (2000), 12-16.

[43] P.S. Tofts and S. Wray, A critical assessment of methods of measuring metabolic concentration by NMR spectroscopy, NMR Biomed. 1 (1988), 1-10.

[44] M.D. Tricklebank, L. Singh, A. Jackson and R.J. Oles, Evidence that a proconvulsant action of lithium is mediated by inhibition of myo-inositol phosphatase in mouse brain, Brain Res. 558 (1991), 145-148.

[45] M.M. Weissman, P.J. Leaf, G.L. Tishler, D.G. Blazer, M. Karno, M.L. Bruce and L.P. Florio, Affective disorders in five United States communities, Psychol. Med. 18 (1988), 141-153.

[46] A. Yildiz, C.M. Demopolus, C.M. Moore, P.F. Renshaw and G.S. Sachs, Effect of lithium on phosphoinositide metabolism in human brain: A proton-decoupled ${ }^{31} \mathrm{P}$ magnetic resonance spectroscopy study, Biol. Psychiatry 50 (2001), 3-7.

[47] A. Yildiz, G.S. Sachs, D.J. Dorer and P.F. Renshaw, ${ }^{31} \mathrm{P}$ Nuclear magnetic resonance spectroscopy findings in bipolar illness: a meta-analysis, Psychiatry Research Neuroimaging 106 (2001), 181-191.

[48] A. Yildiz and Z. Tunca, Recent findings in mechanisms underlying mood stabilizing effects of lithium and valproate, Turkish J. Psychiatry 12 (2001), 147-155.

[49] P.X. Yuan, G. Chen, L.D. Huang and H.K. Manji, Lithium stimulates gene expression through the AP-1 transcription factor pathway, Mol. Brain Res. 58 (1998), 225-230. 


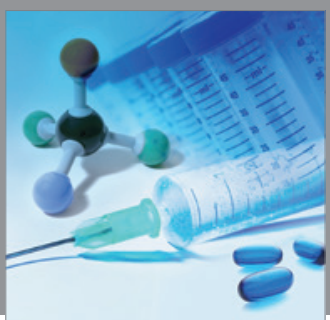

International Journal of

Medicinal Chemistry

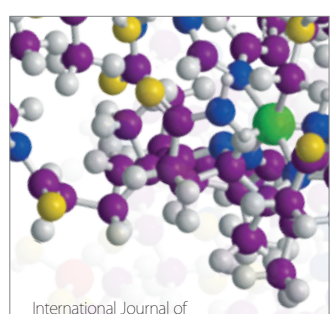

Carbohydrate Chemistry

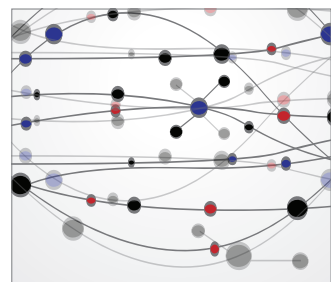

The Scientific World Journal
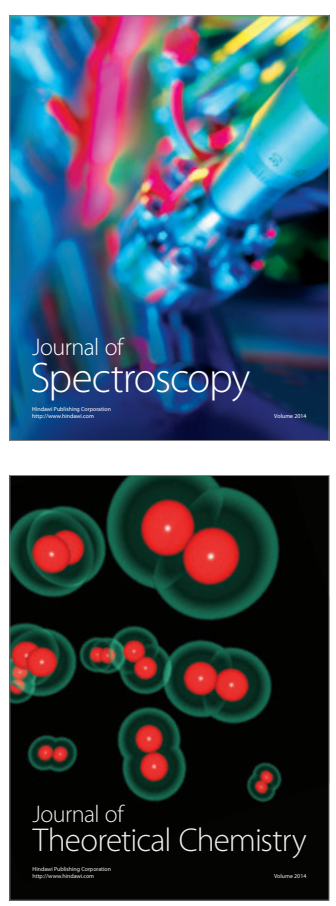
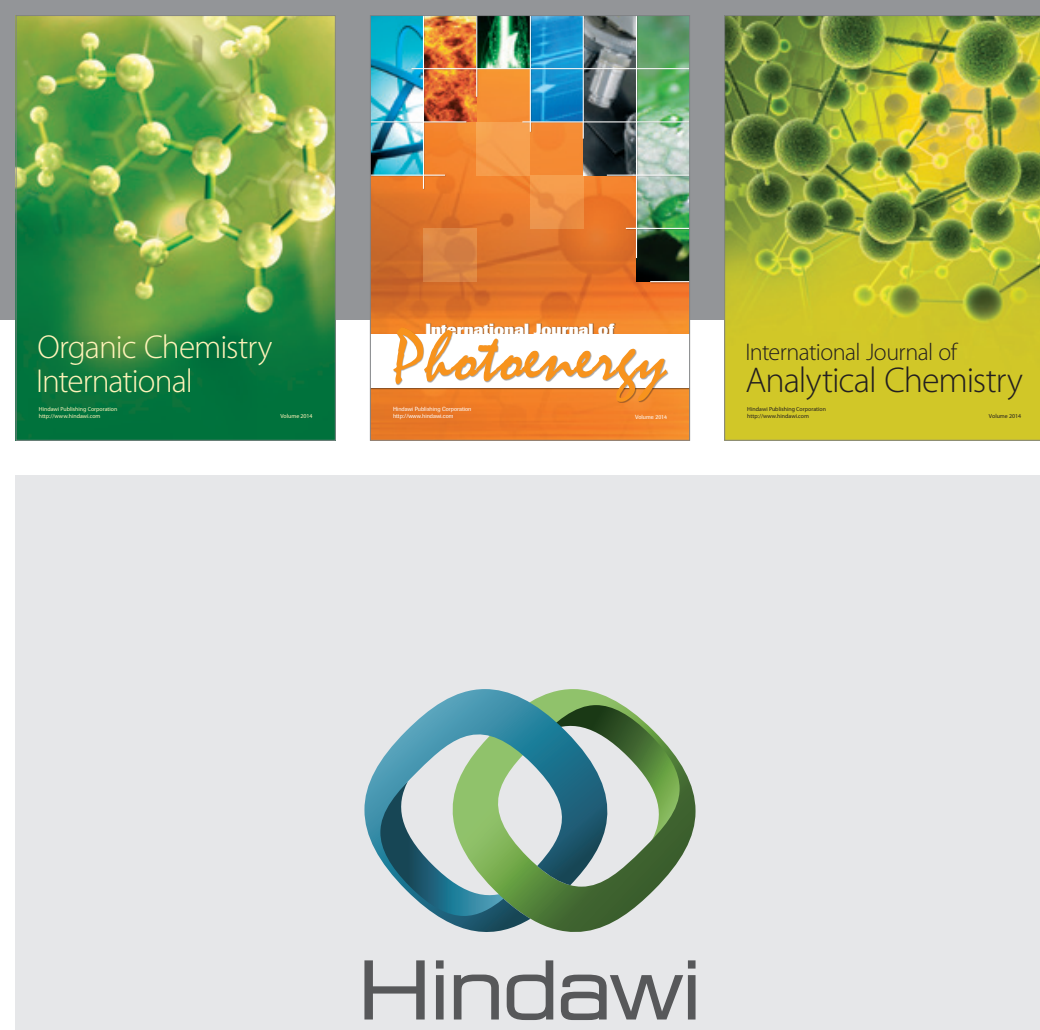

Submit your manuscripts at

http://www.hindawi.com
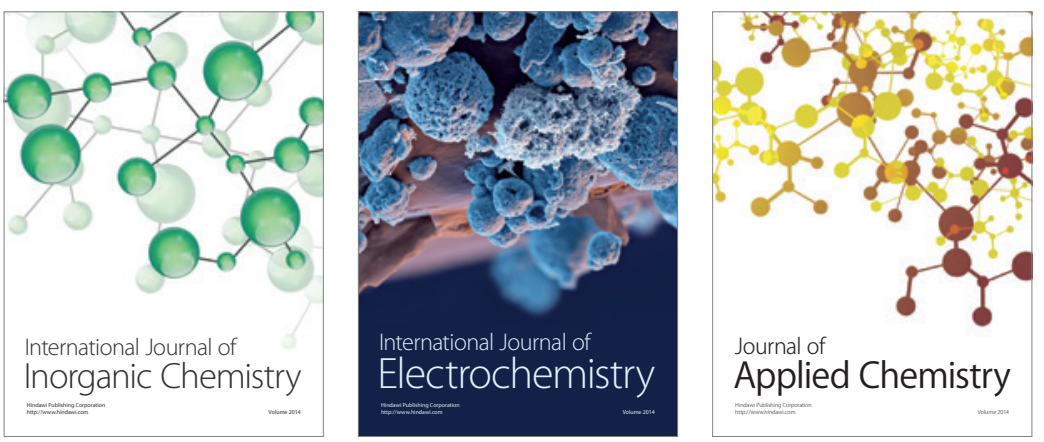

Journal of

Applied Chemistry
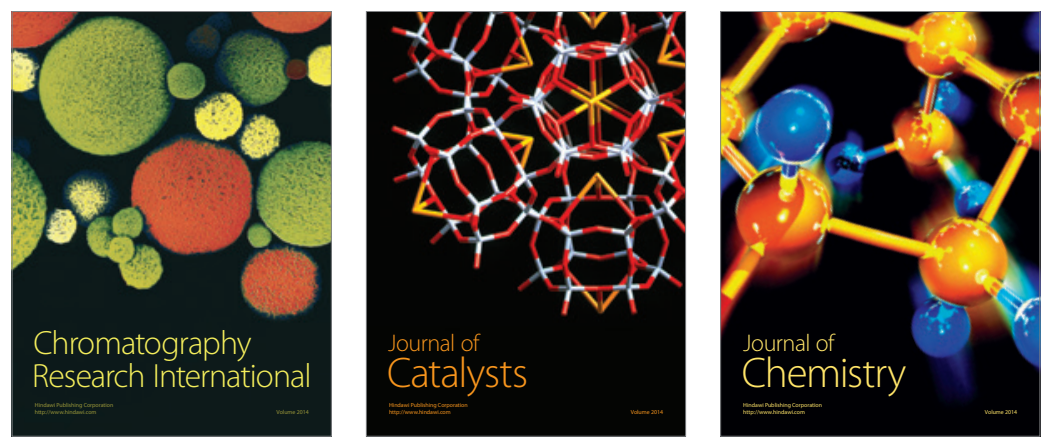
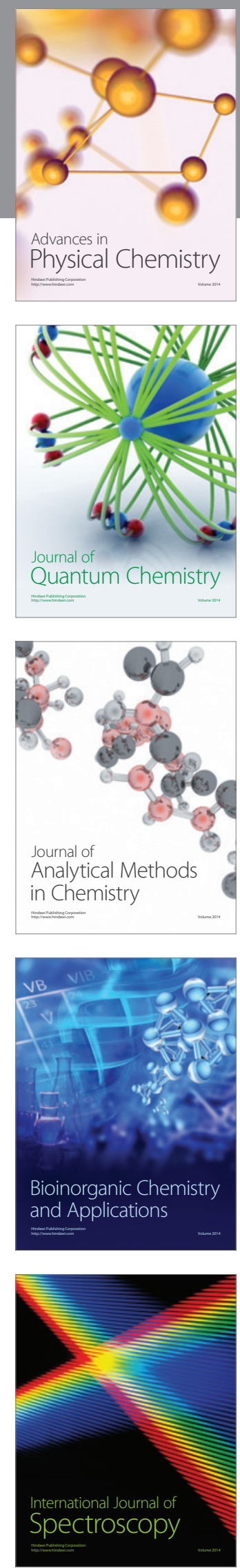